\title{
A ligation-independent cloning method using nicking DNA endonuclease
}

\author{
Jie Yang, Zhihong Zhang, Xin A. Zhang, and Qingming Luo \\ Britton Chance Center for Biomedical Photonics, Wuhan National Laboratory for Optoelectronics-Huazhong \\ University of Science and Technology, Wuban, China
}

BioTechniques 49:817-821 (November 2010) doi 10.2144/000113520

Keywords: ligation-independent cloning (LIC); nicking DNA endonuclease (NiDE); plasmid construction

Supplementary material for this article is available at www.BioTechniques.com/article/113520.

Using nicking DNA endonuclease (NiDE), we developed a novel technique to clone DNA fragments into plasmids. We created a NiDE cassette consisting of two inverted NiDE substrate sites sandwiching an asymmetric four-base sequence, and NiDE cleavage resulted in 14-base single-stranded termini at both ends of the vector and insert. This method can therefore be used as a ligation-independent cloning strategy to generate recombinant constructs rapidly. In addition, we designed and constructed a simple and specific vector from an Escherichia coli plasmid backbone to complement this cloning method. By cloning cDNAs into this modified vector, we confirmed the predicted feasibility and applicability of this cloning method.

Gene cloning and vector construction are widely applied techniques in DNA and protein research. Conventional methods to insert genes into vectors are based on DNA cleavage by restriction endonucleases and then ligation by DNA ligase. However, this DNA engineering method is time-consuming and relatively inefficient. To more efficiently clone DNA molecules, several ligation-independent cloning (LIC) methods have been developed, such as LIC based on T4 DNA polymerase (1-5), GATEWAY recombination $(6,7)$, In-Fusion (8-10), uracil-DNA glycosylase (UDG) (11-13), and sequence- and ligationindependent cloning (SLIC) (14). These methods can be used to construct DNA with high efficiency, but most of them are still time-consuming or expensive. LIC based on T4 DNA polymerase involves assembling DNA molecules by using noncovalent complementation of single-stranded DNA of the insert and vector. Briefly, it relies on 10 - to 15-nucleotide complementary $3^{\prime}$ overhangs at the ends of a PCR-amplified DNA fragment and a linearized vector to make a stable hybridization product that can be readily used to transform host organisms without ligation (5). However, LIC based on T4 DNA polymerase cloning is strictly sequence-dependent because it requires the presence or absence of specific nucleotides at certain positions in the overlapping region (1-5), which restricts its widespread application. T4 DNA polymerase based on SLIC cloning methods are more flexible and sequence-independent, and are the preferred methods so far. However, they are not strictly sequence-independent, because they use in vitro homologous recombination and singlestrand annealing (14). The annealing step in these methods is normally performed at ambient temperature, which allows nonspecific hybridization among single-stranded overhangs and leads to frequent assembly errors (15); its success rate is only $17 \%$ (7 of 42 Escherichia coli transformants) (16). DNA assembler is a much easier method and allows the assembly of an entire biochemical pathway in a single step via in vivo homologous recombination in Saccharomyces cerevisiae (16), but it is much more inefficient in bacteria than it is in yeast. Recently, another LIC methodology adopted UDG to excise the uracil residues that were incorporated into the DNA strand through PCR primers (11-13). This method is also useful for efficiently cloning DNA. However, uracil excision-based cloning needs at least four kinds of tool enzymes, including restrictive endonuclease, UDG, Nt.BbvCI, and DNA glycosylase-lyase endo VIII (13).

To address these limitations, we developed a novel and straightforward LIC method based on nicking DNA endonuclease (NiDE). NiDEs cleave only one strand of DNA on a double-stranded DNA substrate. Nt.BbvCI, a NiDE, recognizes the substrate illustrated in Supplementary Figure S1A. According to this principle, we designed an Nt.BbvCI cassette with two inverted Nt.BbvCI substrate sites with four asymmetric bases between these two sites (Supplementary Figure S1B). When Nt.BbvCI digests this DNA site, the doublestranded DNA can be cleaved to form a 14-nucleotide, single-strand overhang tail. Compared with UDG-LIC methodology, our method shows similar efficiency and lower cost, because it requires only two kinds of enzymes.

\section{Materials and methods}

Vector design

ToconstructaNiDE-compatiblepLIC vector, LacZ cDNA was amplified from a modified pMD18 construct by PCR using primers Laz-BgiII-F and Laz-EcoRI-R (Supplementary Table S1). The $\beta$-galactosidase gene lac $Z$, including restriction endonuclease sites such as BamHI, SalI, PstI, and SphI, was amplified and then digested by $B g l$ II and $E c o$ RI. It was then cloned into pRSETb plasmid between BamHI and Eco RI sites to generate the pLIC vector. 


\section{BioTechnioues \\ The International Journal of Life Science Methods}

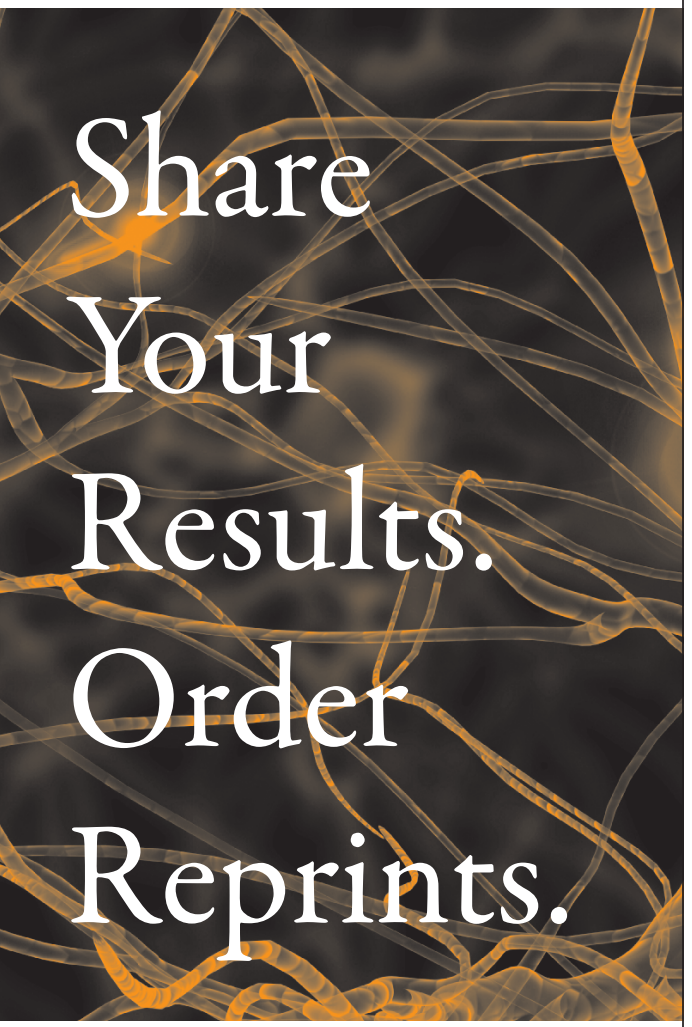

Author Reprints

Make the most of your hard work by ordering reprints of your article published in BioTechniques. Reprints are an inexpensive and easy way to distribute your findings to students and colleagues alike.

\section{Corporate Reprints}

Leverage BioTechniques, the most powerful brand in the market. Reprints help support your sales effort by utilizing articles that spotlight your brand/products to educate customers at meetings and industry trade events.

\section{More info:}

www.BioTechniques.com/advertise/reprints

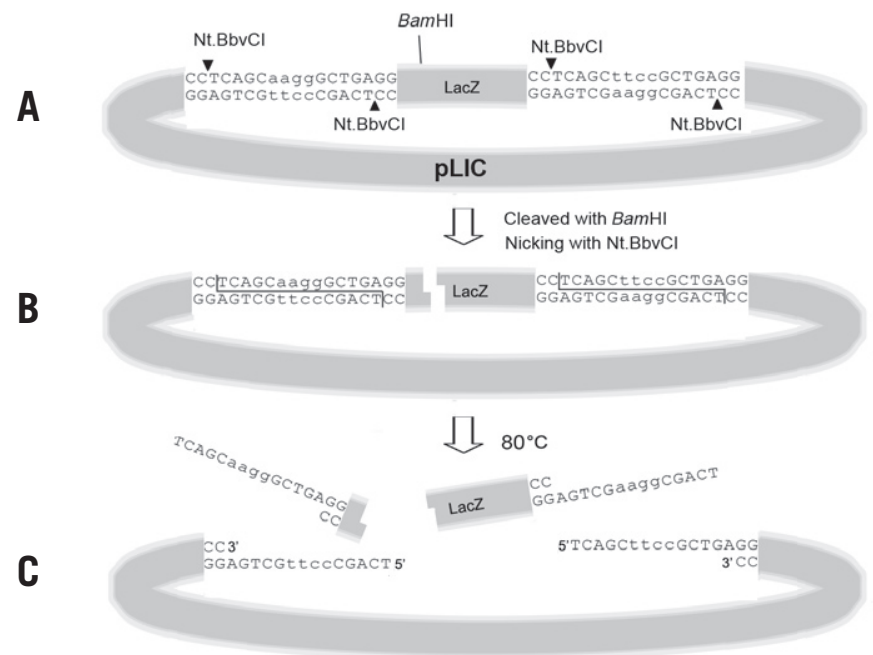

Figure 1. Preparing a vector with long single-strand $5^{\prime}$ overhangs. (A) The framework of the pLIC vector, which is a NiDE-compatible vector. (B) The pLIC vector was digested by $\mathrm{BamHI}$ and $\mathrm{Nt}$. BbvCl to form linearization and nick. (C) Two long single-strand $5^{\prime}$ overhangs in the pLIC vector were produced when temperature increased to $80^{\circ} \mathrm{C}$.

\section{A}

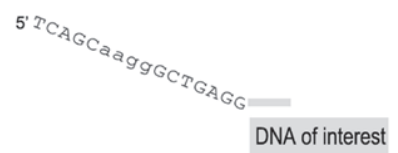

B

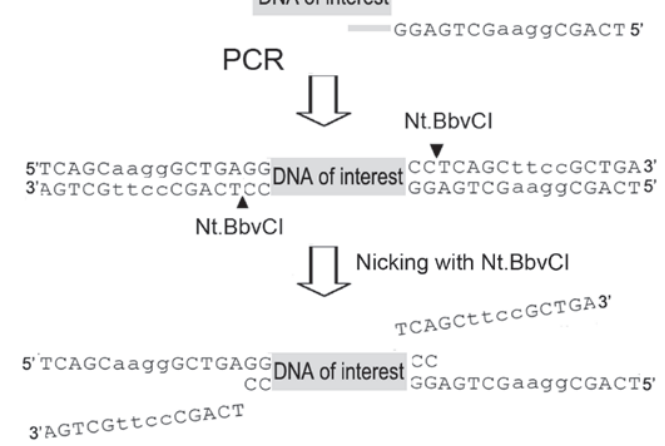

Figure 2. Preparing a DNA fragment with long single-strand 5' overhangs. (A) The DNA of interest was amplified using PCR with primers containing the 16 -nucleotide sequence at the $5^{\prime}$ end and the sequence specific to the target DNA fragment at the $3^{\prime}$ end (see the "Materials and methods" section). (B) PCR fragments are digested by Nt. BbvCl to form two nicks near the $3^{\prime}$ end. (C) Two long singlestrand $5^{\prime}$ overhangs in the PCR fragments were produced when temperature increased to $80^{\circ} \mathrm{C}$.

DNA amplification and assembly

To prepare linear pLIC, $5 \mu \mathrm{g}$ plasmid DNA was digested with $40 \mathrm{U}$ Bam HI in $50 \mu$ L NEB buffer 4 (New England Biolabs, Ipswich, MA, USA) at $37^{\circ} \mathrm{C}$ for $16 \mathrm{~h}$. The reaction mixture was subsequently incubated at $80^{\circ} \mathrm{C}$ for 20 min to inactivate $B a m \mathrm{HI}$ and was then stored at $-20^{\circ} \mathrm{C}$ for further use without purification.

Four PCR amplifications were performed using plasmid templates containing fulllength cDNAs of yellow fluorescent protein Venus (17), red fluorescent protein mLumin (18), bFos (bZIPdomain of cFos) (19), and presenilin 1. DNA fragments to be inserted into the $\mathrm{Nt}$. BbvCI cassette-containing vectors were amplified with PCR primers, which contained the sequence specific to the target DNA fragment and a tail of 16 nucleotides (see below). Forward primer: 5'-TCAGCaaggGCTGAGG-3' plus 5-40 nucleotides complementary sequence to template DNA, reverse primer: 5'-TCAGCggaaGCTGAGG-3' plus 25-40 nucleotides complementary sequence to template DNA (PCR primer sequences are shown in Supplementary Table S1). PCR was performed with high fidelity Pyrobest DNA polymerase (Takara, Dalian, China) according to the manufacturer's instructions. The PCR products were column-purified using PCR purification kit (Tiangen, Beijing, China) according to the manufacturer's instructions. The concentrations of $\mathrm{pLIC}$ and PCR product were measured by using a Biophotometer spectrophotometer (Eppendorf, Hamburg, 
Table 1. Recombinant colony counts using different molar ratios of vector to insert.

\begin{tabular}{|cccc|} 
Molar ratio of vector to insert & White clones & Blue clones & Recombinant colonies (\%) \\
\hline $1: 1$ & 93 & 8 & 92 \\
$1: 2$ & 150 & 5 & 97 \\
$1: 3$ & 207 & 6 & 97 \\
$1: 5$ & 230 & 9 & 96 \\
$1: 10$ & 223 & 7 & 97 \\
\hline
\end{tabular}

Blue-white selection was performed to distinguish the recombinant colonies (white) from the colonies carrying vector background (blue). The molar amount of vector was $0.01 \mathrm{pmol}$ in each reaction. Each count is the average of three repeats.

Table 2. Vector efficiency for inserts of different lengths.

\begin{tabular}{|lcccc|}
\multicolumn{1}{|c}{ LIC insert } & White clones & Blue clones & $\begin{array}{c}\text { Vector Efficiency } \\
\text { (cfu//pg) }\end{array}$ & $\begin{array}{c}\text { Recombinant } \\
\text { colonies (\%) }\end{array}$ \\
\hline bFos (282 bp) & 235 & 7 & $1.17 \times 10^{4}$ & 97 \\
\hline Venus (714 bp) & 230 & 9 & $1.06 \times 10^{4}$ & 96 \\
\hline mLumin (711 bp) & 186 & 7 & $9.3 \times 10^{3}$ & 96 \\
\hline PS1 (1407 bp) & 163 & 10 & $6.65 \times 10^{3}$ & 94 \\
\hline
\end{tabular}

The molar amount of vector was $0.01 \mathrm{pmol}$. Vector mixed with 5 -fold molar PCR fragment. Each count is the average of three repeats. Cell competency of DH5a-competent cells was $2 \times 10^{6} \mathrm{cfu} / \mathrm{\mu g}$ for undigested vector.

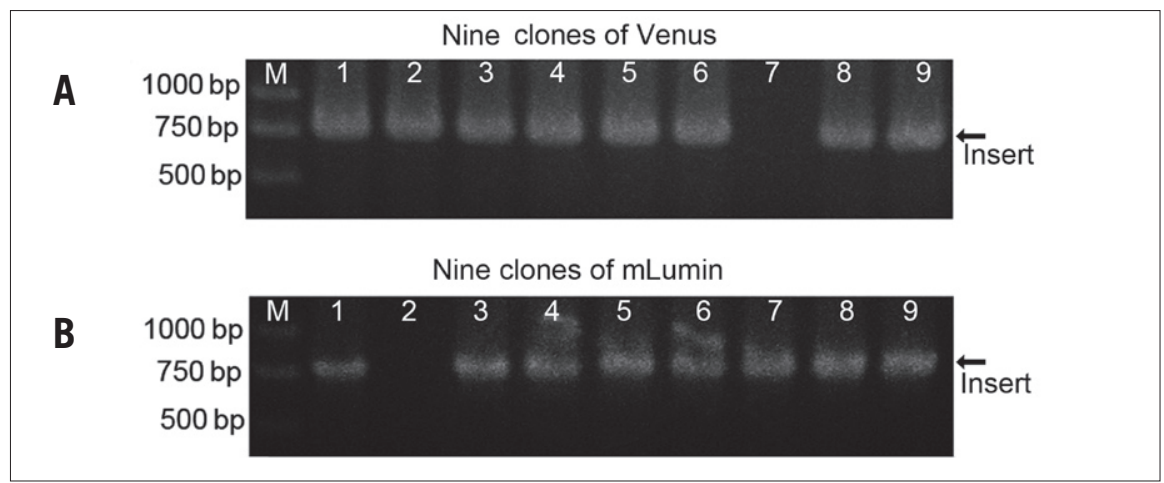

Figure 3. Analysis of potential clones. Plasmids from nine independent Venus colonies $(A)$ and nine mLumin colonies (B) were amplified and run on an agarose gel. A 5- $\mu \mathrm{L}$ aliquot of reaction was separated on a $1 \%$ agarose gel. Lanes 1-9, PCR products of nine colonies; M, DNA marker.

Germany). Purified PCR products were adjusted to $20 \mathrm{ng} / \mu \mathrm{L}$. Different molar ratios of vector to insert ( 1:1-1:10) were mixed with 5 UNt.BbvCI (New England Biolabs) and $2 \mu \mathrm{L}$ NEB buffer 4; the final reaction volume was adjusted to $20 \mu \mathrm{L}$ by adding water. Then $20 \mathrm{ng}$ linearized vector pLIC was added to the reaction mixture, and the nicking reaction proceeded at $37^{\circ} \mathrm{C}$ for $1 \mathrm{~h}$. The mixture was then incubated at $80^{\circ} \mathrm{C}$ for $10 \mathrm{~min}$ and $30^{\circ} \mathrm{C}$ for $10 \mathrm{~min}$ to anneal the vector and insert termini. The reaction mixture was used to transform E. coli-competent cells using the heat-shock method.

\section{Results and discussion}

To generate a NiDE-compatible vector, we inserted the $\alpha$-peptide coding region of lac $Z$ flanked by two Nt.BbvCI cassettes into the multiplecloning site of pRSETbvector to form the pLIC vector (Figure 1). To avoid pLIC self-annealing after $\mathrm{Nt}$. BbvCI digestion, two different $\mathrm{Nt}$. BbvCI cassettes were designed. The forward cassette sequence 5'-CCTCAGCaaggGCTGAGG- $3^{\prime}$ and rear cassette sequence 5'-CCTCAGCttccGCTGAGG-3' were different from each other by 4 bp (the interval sequence is indicated in lowercase). Such a design also permitted controlled orientation of inserts.

To design the NiDE-compatible PCR primer, a tail of 16 nucleotides was inserted at the $5^{\prime}$-terminal of the primer, specific to the target DNA fragment (see the "Materials and methods" section). The 16-nucleotide sequence was complementary to the $\mathrm{Nt}$. BbvCI cassettes in the $\mathrm{pLIC}$ vector. After digestion by Nt.BbvCI, the PCR product formed a 14-nucleotide overhang at the 3 -terminal (Figure 2), which was able to bind with the pLIC vector digested by Nt.BbvCI.

Successful insertion of the PCR product into the vector resulted in displacement of $l a c Z$.
Removing $\beta$-galactosidase allowed recombinant clones to be directly identified by color screening on indicator plates. Since this gene could also insert back into the vector to form clones, there were initially many background clones. To overcome this problem, we used restriction endonuclease Bam $\mathrm{HI}$ to digest lacz, and the number of blue clones decreased significantly (data not shown). After a 16-h digestion, the linearized vector was tested for the digestion ratio by a transformation assay. Transformation results yielded $270 \mathrm{cfu} / \mu \mathrm{g}$ linearized vector compared with $2 \times 10^{6} \mathrm{cfu} / \mu \mathrm{g}$ uncut vector, indicating that only $0.0135 \%$ of the vector remained uncut.

We used the NiDE method to clone Venus (714 bp) by using different molar ratios of vector to insert $(1: 1,1: 2,1: 3,1: 5$, and $1: 10)$. The pLIC vectors generated the most white clones (230) and 96\% recombinant colonies when incubated with a 5 -fold molar ratio of PCR fragment (Table 1).

We also assembled bFos (282 bp), mLumine (711 bp), and PS1 (1407 bp) genes into linear plasmids. The pLIC vectors had a cloning efficiency of approximately 200 colonies per 0.01 pmol vector when incubated with a 5 -fold molar ratio of PCR fragment (Table 2). This result is comparable to the cloning efficiency of USER vector previously reported (12). For verification, we randomly selected nine Venus and nine mLumin white clones and analyzed them with PCR amplification. The percentage of positive clones was $88 \%$ (Figure 3 ).

We also used another nicking DNA endonuclease, $\mathrm{Nb} . \mathrm{BsrDI}$, to clone Venus into the vector, in which two sequences, $5^{\prime}$-NNCATTGCccGCAATGNN-3' and 5'-NNCATTGCtttGCAATGNN-3' (note: NNCATTGC is the $\mathrm{Nb}$. BsrDI recognition sequence), were used for the $\mathrm{Nb}$.BsrDI cassettes. It produced $\sim 40 \%$ false-positive clones. DNA sequencing revealed these clones were mostly self-circularized vector without insert. We speculate that only two or three bases that are inserted between two Nb.BsrDI recognition sequences cannot efficiently prevent the vector's self-circularizing. In contrast, $\mathrm{Nt}$.BbvCI cassettes sandwiching four bases avoided self-circularizing.

When a linear DNA molecule with two or more Nt.BbvCI-recognizing sites is digested by Nt.BbvCI, the digested DNA molecules will be still bonded together at $37^{\circ} \mathrm{C}$ for long complementary sequences, unless the distance of two Nt.BbvCI-recognizing sites is small enough. However, this probability is rare. If two Nt.BbvCI-recognizing sites are separated by a long DNA sequence ( $>30 \mathrm{bp}$ ) with high Tm value, the cleaved DNA can still stay together by a hydrogen bond at $37^{\circ} \mathrm{C}$ after being cleaved by Nt.BbvCI. Accordingly, this method will be very suitable for 
constructing cDNA libraries or large DNA fragments, because it will greatly decrease chances of cleaving the inserts.

In conclusion, using the nicking DNA endonuclease Nt.BbvCI, PCR inserts can assemble into our modified vector with high efficiency. Compared with conventional cloning methods, the NiDE method has several advantages. First, the NiDE cloning method is highly efficient. Second, it saves time because the constructions can be completed in $\sim 2-3 \mathrm{~h}$. Last, the technique is less labor- and cost-intensive since purification of the digested vector and insert is no longer required. Because of these advantages, we expect it to be used for high-throughput plasmid construction.

\section{Acknowledgments}

This work was supported by the National Natural Science Foundation of China (grant nos. 30800208 and 30770525), National High-Tech R\&D Program of China (grant no. 2006AA020801), Program for Changjiang Scholars and Innovative Research Team in University, and the Program for New Century Excellent Talents in University of China (grant no. NCET-080220, to Z.H.Z.).

\section{Competing interests}

The authors declare no competing interests.

\section{References}

1. Tachibana, A., K. Tohiguchi, T. Ueno, Y. Setogawa, A. Harada, and T. Tanabe. 2009. Preparation of long sticky ends for universal ligation-independent cloning: sequential T4 DNA polymerase treatments. J. Biosci. Bioeng. 107:668-669.

2. Weeks, S.D., M. Drinker, and P.J. Loll. 2007. Ligation independent cloning vectors for expression of SUMO fusions. Protein Expr. Purif. 53:40-50.

3. Eschenfeldt, W.H., S. Lucy, C.S. Millard, A. Joachimiak, and I.D. Mark. 2009. A family of LIC vectors for high-throughput cloning and purification of proteins. Methods Mol. Biol. 498:105-115.

4. Bardóczy, V., V. Géczi, T. Sawasaki, Y. Endo, and T. Mészáros. 2008. A set of ligationindependent in vitro translation vectors for eukaryotic protein production. BMC Biotechnol. 27:32.

5. Aslanidis, C., P.J. de Jong, and G. Schmitz. 1994. Minimal length requirement of the single-stranded tails for ligation-independent cloning (LIC) of PCR products. PCR Methods Appl. 4:172-177.

6. Walhout, A.J., G.F. Temple, M.A. Brasch, J.L. Hartley, M.A. Lorson, S. van den Heuvel, and M. Vidal. 2000. GATEWAY recombinational cloning: application to the cloning of large numbers of open reading frames or ORFeomes. Methods Enzymol. 328:575-592.
7. Atanassov, I.I., I.I. Atanassov, J.P. Etchells, and S.R. Turner. 2009. A simple, flexible and efficient PCR-fusion/Gateway cloning procedure for gene fusion, site-directed mutagenesis, short sequence insertion and domain deletions and swaps. Plant Methods 5:14.

8. Berrow, N.S., D. Alderton, and R.J. Owens. 2009. The precise engineering of expression vectors using high-throughput In-Fusion PCR cloning. Methods Mol. Biol. 498:75-90.

9. Zhu, B., G. Cai, E.O. Hall, and G.J. Freeman. 2007. In-Fusion assembly: seamless engineering of multidomain fusion proteins, modular vectors, and mutations. BioTechniques 43:354-359.

10. Berrow, N.S., D. Alderton, S. Sainsbury, J. Nettleship, R. Assenberg, N. Rahman, D.I. Stuart, and R.J. Owens. 2007. A versatile ligation-independent cloning method suitable for high-throughput expression screening applications. Nucleic Acids Res. 35:e45.

11. Bitinaite, J., M. Rubino, K.H. Varma, I. Schildkraut, R. Vaisvila, and R. Vaiskunaite. 2007. USER friendly DNA engineering and cloning method by uracil excision. Nucleic Acids Res. 35:1992-2002.

12. Geu-Flores, F., H.H. Nour-Eldin, M.T. Nielsen, and B.A. Halkier. 2007. USER fusion: a rapid and efficient method for simultaneous fusion and cloning of multiple PCR products. Nucleic Acids Res. 35:e55.

13. Nour-Eldin, H.H., B.G. Hansen, M.H. Nørholm, J.K. Jensen, and B.A. Halkier. 2006. Advancing uracil-excision based cloning towards an ideal technique for cloning PCR fragments. Nucleic Acids Res. 34:e122.

14. Li, M.Z. and S.J. Elledge. 2007. Harnessing homologous recombination in vitro to generate recombinant DNA via SLIC. Nat. Methods 4:251-256.

15. Quan, J. and J. Tian. 2009. Circular polymerase extension cloning of complex gene libraries and pathways. PLoS One 4:e6441.

16. Shao, Z., H. Zhao, and H. Zhao. 2009. DNA assembler, an in vivo genetic method for rapid construction of biochemical pathways. Nucleic Acids Res. 37:e16.

17. Nagai, T., K. Ibata, E.S. Park, M. Kubota, K. Mikoshiba, and A. Miyawaki. 2002. A variant of yellow fluorescent protein with fast and efficient maturation for cell-biological applications. Nat. Biotechnol. 20:87-90.

18. Chu, J., Z. Zhang, Y. Zheng, J. Yang, L. Qin, J. Lu, Z.L. Huang, S. Zeng, and Q. Luo. 2009. A novel far-red bimolecular fluorescence complementation system that allows for efficient visualization of protein interactions under physiological conditions. Biosens. Bioelectron. 25:234-239.

19. Shyu, Y.J., C.D. Suarez, and C. Hu. 2008. Visualization of AP-1-NF- $\kappa$ B ternary complexes in living cells by using a BiFC-based FRET. Proc. Natl. Acad. Sci. USA 105:151-156.

Received 26 May 2010; accepted 30 August 2010.

Address correspondence to Zhihong Zhang, Britton Chance Center for Biomedical Photonics, Wuhan National Laboratory for OptoelectronicsHuazhong University of Science and Technology, Wuhan 430074, China. e-mail: czyzzh@mail. hust.edu.cn

To purchase reprints of this article, contact: carmelitag@fosterprinting.com 